\title{
Inner Products Involving Differences: The Meixner-Sobolev Polynomials
}

\author{
I. AREA ${ }^{a}, E, G O D O Y^{b} * *$ and F. MARCELLÁN ${ }^{c}$ \\ a Departamento de Matemática Aplicada, E.T.S.E. Telecomunicación, \\ b Departamento de Matemática Aplicada, E.T.S.I.Industriales y Minas, \\ Universidad de Vigo, Campus Lagoas-Marcosende, 36200 Vigo, Spain; \\ 'Departamento de Matemáticas, Escuela Politécnica Superior, Universidad \\ Carlos III de Madrid, c/Butarque 15, 28911-Leganés, Madrid, Spain
}

In this paper, polynomials which are orthogonal with respect to the inner product

$$
\langle p, q\rangle_{\mathrm{S}}=\sum_{s=0}^{\infty} p(s) q(s) \frac{\mu^{s} \Gamma(\gamma+s)}{\Gamma(s+1) \Gamma(\gamma)}+\lambda \sum_{s=0}^{\infty} \Delta p(s) \Delta q(s) \frac{\mu^{s} \Gamma(\gamma+s)}{\Gamma(s+1) \Gamma(\gamma)}
$$

where $0<\mu<1, \gamma>0$ and $\lambda \geq 0$ are studied. For these polynomials, algebraic properties and difference equations are obtained as well as their relation with the Meixner polynomials. Moreover, some properties about the zeros of these polynomials are deduced.

Keywords: Meixner polynomials; Sobolev orthogonal polynomials; Difference operators; Pollaczek polynomials; Zeros of orthogonal polynomials; Polynomial approximation

Classification Category: MSC 33C25, 33D25, 39A10, 39A70, 42C05

*Corresponding author. E-mail: egodoy@dma.uvigo.es. 


\section{INTRODUCTION}

Let $\mathbb{P}$ be the linear space of polynomials with real coefficients. If we define an inner product on $\mathbb{P}$

$$
(f, g)=\int_{\mathbb{R}} f(x) g(x) \mathrm{d} \mu(x),
$$

where $\mathrm{d} \mu(x)$ is a signed measure on the real line; it is known (see [5, pp. 21-22]) that there exists a sequence of polynomials $\left\{P_{n}(x)\right\}_{n}$ such that

$$
\begin{aligned}
\operatorname{deg} P_{n} & =n, \\
\left(P_{n}, P_{m}\right) & =k_{n} \delta_{n m}, \quad k_{n} \neq 0 .
\end{aligned}
$$

We assume that the sequence $\left\{P_{n}(x)\right\}_{n}$ is monic, i.e. the leading coefficient of $P_{n}(x)$ is one. In these conditions, the sequence $\left\{P_{n}(x)\right\}_{n}$ is called a Monic Orthogonal Polynomial Sequence (MOPS) with respect to the inner product (1). Such a MOPS $\left\{P_{n}(x)\right\}_{n}$ satisfies a three-term recurrence relation

$$
\begin{aligned}
& P_{n+1}(x)=\left(x-B_{n}\right) P_{n}(x)-C_{n} P_{n-1}(x), \quad n \geq 0 \\
& P_{0}(x)=1, \quad P_{-1}(x)=0, \quad C_{n} \neq 0, \quad n=1,2, \ldots
\end{aligned}
$$

The original motivation for considering Sobolev orthogonal polynomials comes from the least squares approximation problems $[10,15]$. A given function $f$ and its derivative $f^{\prime}$ are to be approximated simultaneously by a polynomial $p$ of degree $n$ minimizing

$$
\|p(x)-f(x)\|^{2}=\int_{\mathbb{R}}[p(x)-f(x)]^{2} \mathrm{~d} \mu_{0}(x)+\lambda \int_{\mathbb{R}}\left[p^{\prime}(x)-f^{\prime}(x)\right]^{2} \mathrm{~d} \mu_{1}(x)
$$

over all $p \in \mathbb{P}_{n}, \mathrm{~d} \mu_{i}(x), i=0,1$, being positive Borel measures on the real line $\mathbb{R}$ having bounded or unbounded support $[8,19]$. Expanding $p$ in terms of the Sobolev orthogonal polynomials we obtain the usual Fourier approximation $p(x)$ of $f(x)$ and $f^{\prime}(x)$. This problem was considered in [15], but nothing was said there about the sequence of 
polynomials $\left\{Q_{n}(x)\right\}_{n}$ orthogonal with respect to the inner product

$$
(f, g)_{\mathrm{S}}=\int_{\mathbb{R}} f(x) g(x) \mathrm{d} \mu_{0}(x)+\lambda \int_{\mathbb{R}} f^{\prime}(x) g^{\prime}(x) \mathrm{d} \mu_{1}(x), \quad \lambda \geq 0 .
$$

Study of polynomials orthogonal with respect to (3), called nondiscrete or continuous case, can be found in $[6,7,19,23]$. Other kind of modifications of (1) are studied in $[1,16,20]$. A new attempt to the study of the non-discrete case was made in 1991 by Iserles et al. [11]. There the authors proved that if the Borel measures $\mathrm{d} \mu_{0}$ and $\mathrm{d} \mu_{1}$ obey a specific condition (coherent pair) then the sequence of orthogonal polynomials $\left\{p_{n}^{(\lambda)}(x)\right\}_{n}$ with respect to (3) can be expanded in terms of the polynomials orthogonal with respect to $\mathrm{d} \mu_{0}$ in such a way that, taking into account an adequate normalization, the expansion coefficients, up to the leading one, are independent of $n$ and are themselves orthogonal polynomials in $\lambda$. They also explored several examples and showed how their theory can be used for an efficient evaluation of Sobolev-Fourier coefficients.

The concept of coherent pair, initialiy defined for measures in [11] can be characterized in terms of the MOPSs $\left\{P_{n}(x)\right\}_{n}$ and $\left\{T_{n}(x)\right\}_{n}$ associated with $\mathrm{d} \mu_{0}$ and $\mathrm{d} \mu_{1}$, respectively [11, Theorem 3] in the following way: there exists a sequence of non-zero complex numbers $\left\{\sigma_{n}\right\}_{n}$ such that

$$
T_{n}(x)=\frac{P_{n+1}^{\prime}(x)}{n+1}-\sigma_{n} \frac{P_{n}^{\prime}(x)}{n}
$$

This concept has been extensively studied by several authors $[17,18,22]$ and it has been recently adapted to the case of orthogonal polynomials of a discrete variable in [2], characterizing the MOPS $\left\{P_{n}(x)\right\}_{n}$ and $\left\{T_{n}(x)\right\}_{n}$ such that

$$
T_{n}(x)=\frac{\Delta P_{n+1}(x)}{n+1}-\sigma_{n} \frac{\Delta P_{n}(x)}{n}
$$

where $\left\{\sigma_{n}\right\}_{n}$ is a sequence of complex numbers and $\Delta$ stands for the forward difference operator $(\Delta h(x)=h(x+1)-h(x))$.

In order to find the best polynomial approximation $p(x)$ of a function $f(x)$ where besides function values $f\left(x_{i}\right)$, also difference 
derivatives at the knots are given, the following minimization problem appears in a natural way:

$\min \sum_{k=0}^{r}\left(\sum_{x_{s}=a_{k}}^{b_{k}-k-1}\left(\Delta^{k} p\left(x_{s}\right)-\Delta^{k} f\left(x_{s}\right)\right)^{2} \rho_{k}\left(x_{s}\right)\right), \quad \Delta^{k} h(x)=\Delta^{k-1}(\Delta h(x))$,

where $\rho_{k}(x)$ are discrete weight functions on $\left[a_{k}, b_{k}\right)$, i.e., each $\rho_{k}(x)$ is piecewise constant function with jumps $\rho_{k}\left(x_{i}\right)$ at the points $x=x_{i}$ for which $x_{i+1}=x_{i}+1$ and $a_{k} \leq x_{i} \leq b_{k}-1$.

Thus, it seems to be interesting the analysis of the polynomials which are orthogonal with respect to the inner product

$$
\langle p, q\rangle_{\mathrm{W}}=\sum_{k=0}^{r}\left(\sum_{x_{s}=a_{k}}^{b_{k} \cdots k-1} \Delta^{k} p\left(x_{s}\right) \Delta^{k} q\left(x_{s}\right) \rho_{k}\left(x_{s}\right)\right) .
$$

The aim of this paper is the study of polynomials which are orthogonal with respect to a particular case $\left(r=1, \rho_{0} \equiv \rho_{1}\right)$ of the above inner product:

$$
\langle p, q\rangle_{\mathrm{S}}=\sum_{s=0}^{\infty} p(s) q(s) \rho(s)+\lambda \sum_{s=0}^{\infty} \Delta p(s) \Delta q(s) \rho(s) ;
$$

$\lambda \geq 0$ and $\rho(s)$ is the Meixner weight function [24]. We call (4) the Meixner-Sobolev inner product, by analogy with the continuous case [21].

The structure of the paper is as follows: Section 2 contains the basic relations for monic Meixner orthogonal polynomials $\left\{M_{n}^{(\gamma, \mu)}(x)\right\}_{n}$. In Section 3, we introduce the Meixner-Sobolev inner product, the monic Meixner-Sobolev orthogonal polynomials $\left\{Q_{n}(x)\right\}_{n}$ and the limit polynomials $\left\{R_{n}(x)\right\}_{n}$ obtained from $\left\{Q_{n}(x)\right\}_{n}$ when $\lambda$ tends to infinity. We also give some relations among these three families of polynomials and a limit relation between Meixner-Sobolev and Laguerre-Sobolev polynomials. In Section 4, a linear difference operator $\mathcal{S}$ on $\mathbb{P}$ is defined. We prove it is a symmetric operator with respect to the Meixner-Sobolev inner product and we find a nonstandard four-term recurrence relation for the $\left\{Q_{n}(x)\right\}_{n}$ polynomials. Finally, in Section 5, we study the properties of the zeros of MeixnerSobolev orthogonal polynomials. 


\section{MONIC MEIXNER ORTHOGONAL POLYNOMIALS}

The forward difference operator $\Delta$ and the backward difference operator $\nabla$ are defined by

$$
\Delta f(x)=f(x+1)-f(x), \quad \nabla f(x)=f(x)-f(x-1) .
$$

These difference operators satisfy the following properties which will be useful in the next sections:

$$
\begin{gathered}
\Delta=\nabla+\Delta \nabla, \quad \Delta p(x)=\nabla p(x+1), \\
\Delta(p(x) q(x))=q(x) \Delta p(x)+p(x+1) \Delta q(x) .
\end{gathered}
$$

Monic Meixner orthogonal polynomials $\left\{M_{n}^{(\gamma, \mu)}(x)\right\}_{n}$ are the polynomial solution of a second order linear difference equation of hypergeometric type $[9,25]$

$$
\begin{gathered}
\sigma(x) \Delta \nabla y(x)+\tau(x) \Delta y(x)+\lambda_{n} y(x)=0, \\
\sigma(x)=x, \quad \tau(x)=\gamma \mu-x(1-\mu), \quad \lambda_{n}=n(1-\mu) .
\end{gathered}
$$

These polynomials $\left\{M_{n}^{(\gamma, \mu)}(x)\right\}_{n}$ are orthogonal with respect to the inner product

$$
\begin{aligned}
& \langle p(x), q(x)\rangle=\sum_{s=0}^{\infty} p(s) q(s) \rho(s), \quad \rho(s)=\frac{\mu^{s} \Gamma(\gamma+s)}{\Gamma(s+1) \Gamma(\gamma)}, \\
& s \in[0,+\infty), \quad 0<\mu<1, \quad \gamma>0 .
\end{aligned}
$$

For monic Meixner orthogonal polynomials the following properties are known $[3,9,25]$.

\subsection{Three-term Recurrence Relation}

We have

$$
\begin{gathered}
x M_{n}^{(\gamma, \mu)}(x)=M_{n+1}^{(\gamma, \mu)}(x)+B_{n} M_{n}^{(\gamma, \mu)}(x)+C_{n} M_{n-1}^{(\gamma, \mu)}(x), \quad n \geq 1, \\
B_{n}=\frac{\gamma \mu+n(1+\mu)}{1-\mu}, \quad C_{n}=\frac{\mu n(\gamma+n-1)}{(1-\mu)^{2}},
\end{gathered}
$$

with the initial conditions $M_{0}^{(\gamma, \mu)}(x)=1, M_{1}^{(\gamma, \mu)}(x)=x-B_{0}$. 


\subsection{Difference Representation}

We have

$$
M_{n}^{(\gamma, \mu)}(x)=\frac{\Delta M_{n+1}^{(\gamma, \mu)}(x)}{n+1}+\frac{\mu}{1-\mu} \Delta M_{n}^{(\gamma, \mu)}(x), \quad n \geq 0 .
$$

\subsection{Representation as Hypergeometric Function}

We have

$$
M_{n}^{(\gamma, \mu)}(x)=\left(\frac{\mu}{\mu-1}\right)^{n}(\gamma)_{n}{ }_{2} F_{1}\left(-n,-x ; \gamma ; 1-\frac{1}{\mu}\right)
$$

where $(a)_{s}$ denotes the Pochhammer symbol, $(a)_{0}=1, \quad(a)_{s}=$ $a(a+1) \cdots(a+s-1)$. From the above hypergeometric representation of monic Meixner polynomials we get

$$
M_{n}^{(\gamma, \mu)}(0)=\left(\frac{\mu}{\mu-1}\right)^{n}(\gamma)_{n}, \quad n \geq 0
$$

\subsection{Squared Norm}

Let us denote

$$
\begin{aligned}
k_{n} & =\left\langle M_{n}^{(\gamma, \mu)}(x), M_{n}^{(\gamma, \mu)}(x)\right\rangle=\sum_{s=0}^{\infty}\left(M_{n}^{(\gamma, \mu)}(s)\right)^{2} \frac{\mu^{s} \Gamma(\gamma+s)}{s ! \Gamma(\gamma)} \\
& =\frac{n !(\gamma)_{n} \mu^{n}}{(1-\mu)^{2 n+\gamma}}, \quad n \geq 0
\end{aligned}
$$

The following relations can be easily derived from the definition of $k_{n}$ :

$$
k_{0}=\frac{1}{(1-\mu)^{\gamma}}, \quad k_{n}=\frac{(\gamma+n-1) \mu n}{(1-\mu)^{2}} k_{n-1}, \quad n \geq 1
$$




\section{MEIXNER-SOBOLEV ORTHOGONAL POLYNOMIALS}

Let us consider the Sobolev inner product defined on $\mathbb{P}$ by

$$
\begin{aligned}
\langle p(x), q(x)\rangle_{\mathrm{S}} & =\langle p(x), q(x)\rangle+\lambda\langle\Delta p(x), \Delta q(x)\rangle \\
& =\sum_{s=0}^{\infty} p(s) q(s) \frac{\mu^{s} \Gamma(\gamma+s)}{s ! \Gamma(\gamma)}+\lambda \sum_{s=0}^{\infty} \Delta p(s) \Delta q(s) \frac{\mu^{s} \Gamma(\gamma+s)}{s ! \Gamma(\gamma)}
\end{aligned}
$$

where $0<\mu<1, \gamma>0$ and $\lambda \geq 0$.

We shall denote by $\left\{Q_{n}^{(\gamma, \mu)}(x ; \lambda)\right\}_{n} \equiv\left\{Q_{n}(x)\right\}_{n}$ the MOPS associated with the inner product $\langle\ldots\rangle_{\mathrm{S}}$. Such a sequence is said to be the Meixner-Sobolev MOPS.

Let us denote the moments associated with the inner product (8) for the basis $\left\{x^{[n]}\right\}_{n}$ as

$$
u_{i, j}=\left\langle x^{[i]}, x^{[j]}\right\rangle=\sum_{s=0}^{\infty} s^{[i]} s^{[j]} \rho(s)=\sum_{s=\max \{i, j\}}^{\infty} s^{[i]} s^{[j]} \rho(s)
$$

with $\rho(x)$ defined in Eq. (8), $x^{[n]}=x(x-1) \cdots(x-n+1), x^{[0]}=1$, and let us denote the moments associated with the inner product (15) for the basis $\left\{x^{[n]}\right\}_{n}$ as $c_{i, j}=\left\langle x^{[i]}, x^{[j]}\right\rangle_{\mathrm{S}}$. Since $\Delta x^{[n]}=n x^{[n-1]}$, we get

$$
\begin{aligned}
& c_{i, 0}=c_{i}=\left\langle x^{[i]}, 1\right\rangle_{\mathrm{S}}=u_{i, 0}=u_{0, i} \equiv u_{i}, \\
& c_{i, j}=\left\langle x^{[i]}, x^{[j]}\right\rangle_{\mathrm{S}}=u_{i, j}+\lambda i j u_{i-1, j-1}, \quad i, j \geq 1 .
\end{aligned}
$$

From the definition,

$$
Q_{0}(x)=M_{0}^{(\gamma, \mu)}(x)=1, \quad Q_{1}(x)=M_{1}^{(\gamma, \mu)}(x)=x-\frac{\gamma \mu}{1-\mu}
$$

but if $\lambda>0$ the elements of these sequences are different for degrees greater than or equal to 2 . 
We can write the Meixner-Sobolev polynomials in the following determinantal form:

$$
\begin{aligned}
& Q_{n}(x)
\end{aligned}
$$

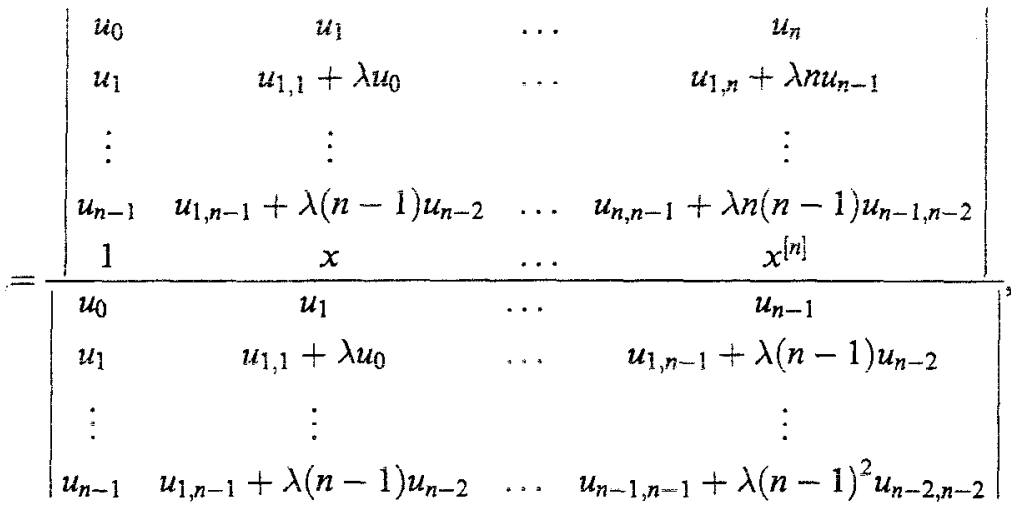

where each coefficient of $Q_{n}(x)$ in terms of $x^{[j]}$ is a rational function in $\lambda$, the numerator and the denominator being of degree $n-1$. Then, we can define a new sequence of monic polynomials $\left\{R_{n}^{(\gamma, \mu)}(x)\right\}_{n} \equiv$ $\left\{R_{n}(x)\right\}_{n}$,

$$
R_{0}(x)=Q_{0}(x)=M_{0}^{(\gamma, \mu)}(x)=1, \quad R_{1}(x)=Q_{1}(x)=M_{1}^{(\gamma, \mu)}(x)
$$

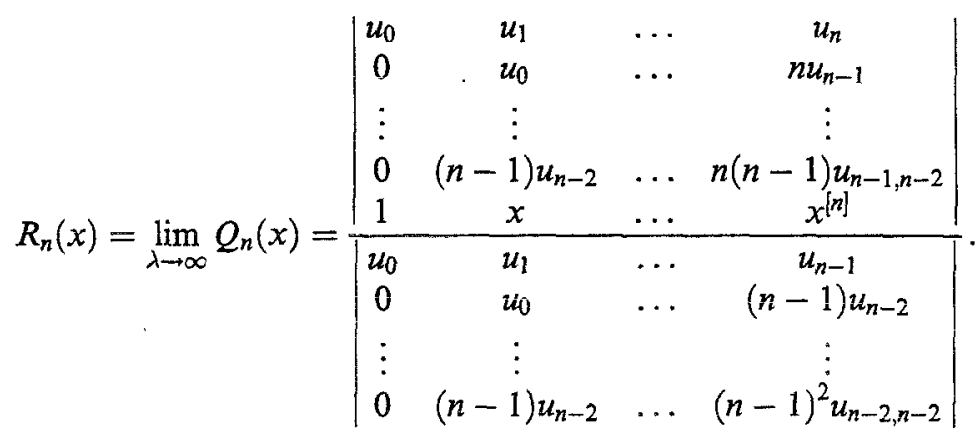


Proposition 1 (a) For each $n \geq 1$ we have $\sum_{s=0}^{\infty} R_{n}(s) \rho(s)=0$, where the weight $\rho(s)$ is given in (8) and the polynomials $R_{n}(x)$ are defined in (19) and (20).

(b) If $n \geq 2$ and $0 \leq m \leq n-2$ then $\sum_{s=0}^{\infty} s^{[m]} \Delta R_{n}(s) \rho(s)=0$, where the polynomials $R_{n}(x)$ are defined in Eqs. (19) and (20), and $\rho(s)$ is given in (8).

Proof

(a) $\sum_{s=0}^{\infty} R_{n}(s) \rho(s)=\sum_{s=0}^{\infty} \lim _{\lambda \rightarrow \infty} Q_{n}(s) \rho(s)=\lim _{\lambda \rightarrow \infty}\left\langle Q_{n}(x), 1\right\rangle_{\mathrm{S}}=0$.

(b) Apply the $\Delta$ operator in the definition of $R_{n}(x)$.

COROLLARY 1 The following two equalities hold:

$$
\begin{gathered}
\Delta R_{n}(x)=n M_{n-1}^{(\gamma, \mu)}(x), \quad n \geq 1 ; \\
R_{n}(x)=M_{n}^{(\gamma, \mu)}(x)+n \frac{\mu}{1-\mu} M_{n-1}^{(\gamma, \mu)}(x) \\
=M_{n}^{(\gamma, \mu)}(x)+\frac{\mu}{1-\mu} \Delta R_{n}(x), \quad n \geq 2 .
\end{gathered}
$$

Remark 1 If $\gamma>1$, then $\left\{R_{n}(x)\right\}_{n} \equiv\left\{M_{n}^{(\gamma-1, \mu)}(x)\right\}_{n}$, i.e., $R_{n}(x)$ is the monic Meixner polynomial of degree $n$ associated with the weight function

$$
\rho^{(\gamma-1, \mu)}(x)=\frac{\mu^{x} \Gamma(x+\gamma-1)}{\Gamma(x+1) \Gamma(\gamma-1)} .
$$

If $0<\gamma \leq 1, R_{n}(x)$ is a quasi-orthogonal polynomial [5, p. 64] of order one with respect to the MOPS $\left\{M_{n}^{(\gamma, \mu)}(x)\right\}_{n}$.

PROPOSITION 2 The following relation holds:

$$
R_{n}(x)=Q_{n}(x)+d_{n-1}(\lambda) Q_{n-1}(x), \quad n \geq 2,
$$

where

$$
\begin{aligned}
d_{n-1}(\lambda) & =n \frac{\mu}{1-\mu} \frac{k_{n-1}}{\tilde{k}_{n-1}}, \quad n \geq 2 \\
\tilde{k}_{n} & =\left\langle Q_{n}(x), Q_{n}(x)\right\rangle_{\mathrm{s}}
\end{aligned}
$$


Proof If $n \geq 2$ we can expand the polynomial $R_{n}(x)$ in terms of Meixner-Sobolev polynomials in the following way:

$$
R_{n}(x)=Q_{n}(x)+\sum_{i=0}^{n-1} f_{i, n}(\lambda) Q_{i}(x)
$$

By using (21) and (22) the coefficients $f_{i, n}(\lambda)$ can be computed as

$$
\begin{aligned}
f_{i, n}(\lambda) & =\frac{\left\langle R_{n}, Q_{i}\right\rangle_{S}}{\left\langle Q_{i}, Q_{i}\right\rangle_{\mathrm{S}}} \\
& =\frac{1}{\tilde{k}_{i}}\left\{\sum_{s=0}^{\infty} R_{n}(s) Q_{i}(s) \rho(s)+\lambda \sum_{s=0}^{\infty} \Delta R_{n}(s) \Delta Q_{i}(s) \rho(s)\right\} \\
& =\frac{1}{\tilde{k}_{i}}\left\{\sum_{s=0}^{\infty} R_{n}(s) Q_{i}(s) \rho(s)+\lambda \sum_{s=0}^{\infty} n M_{n-1}^{(\gamma, \mu)}(s) \Delta Q_{i}(s) \rho(s)\right\} \\
& =\frac{1}{\tilde{k}_{i}} \sum_{s=0}^{\infty}\left\{M_{n}^{(\gamma, \mu)}(s)+n \frac{\mu}{1-\mu} M_{n-1}^{(\gamma, \mu)}(s)\right\} Q_{i}(s) \rho(s) \\
& =\frac{1}{\tilde{k}_{i}}\left\langle M_{n}^{(\gamma, \mu)}(x), Q_{i}(x)\right\rangle+\frac{n \mu}{(1-\mu) \tilde{k}_{i}}\left\langle M_{n-1}^{(\gamma, \mu)}(x), Q_{i}(x)\right\rangle \\
0 & \leq i \leq n-1 .
\end{aligned}
$$

Thus,

$$
f_{i, n}(\lambda)= \begin{cases}0 & \text { if } 0 \leq i \leq n-2 \\ \frac{n \mu}{1-\mu} \frac{k_{i}}{\tilde{k}_{i}} & \text { if } i=n-1\end{cases}
$$

Then (26) becomes

$$
R_{n}(x)=Q_{n}(x)+\frac{n \mu}{1-\mu} \frac{k_{n-1}}{\tilde{k}_{n-1}} Q_{n-1}(x), \quad n \geq 2
$$

COROLLARY 2 The Meixner-Sobolev orthogonal polynomials defined in (18) satisfy

$$
Q_{n}(x)=\sum_{j=1}^{n} e_{j, n} M_{j}^{(\gamma, \mu)}(x), \quad n \geq 2
$$


where

$$
\begin{aligned}
& e_{n, n}=1, \\
& e_{j, n}=(-1)^{n-j-1}\left[\frac{\mu}{1-\mu}(j+1)-d_{j}(\lambda)\right] \prod_{s=j+1}^{n-1} d_{s}(\lambda), \quad 1 \leq j \leq n-1 .
\end{aligned}
$$

Proof From Eqs. (23) and (22), we obtain

$$
Q_{n}(x)=M_{n}^{(\gamma, \mu)}(x)+n \frac{\mu}{1-\mu} M_{n-1}^{(\gamma, \mu)}(x)-d_{n-1}(\lambda) Q_{n-1}(x), \quad n \geq 2,
$$

where repeating this substitution the result follows taking into account that $Q_{1}(x)=M_{1}^{(\gamma, \mu)}(x)$.

We can compute recursively the coefficients $d_{n}(\lambda)$ defined in (24) by means of

Proposition 3 The coefficients $d_{n}(\lambda)$ satisfy the following two-term recurrence relation:

$$
\begin{aligned}
& d_{n}(\lambda) \\
& \quad=\frac{\mu^{2}(n+1)(\gamma+n-1)}{(1-\mu)\left\{\mu(\gamma+n-1)+n\left(\mu^{2}+\lambda(1-\mu)^{2}\right)-\mu(1-\mu) d_{n-1}(\lambda)\right\}},
\end{aligned}
$$

valid for $n \geq 2$, with the initial condition

$$
d_{1}(\lambda)=\frac{2 \gamma \mu^{2}}{(1-\mu)\left(\gamma \mu+\lambda(\mu-1)^{2}\right)}
$$


Proof From (25) and using (11) we get

$$
\begin{aligned}
\tilde{k}_{n}= & \left\langle Q_{n}(x), M_{n}^{(\gamma, \mu)}(x)\right\rangle_{\mathrm{S}}=k_{n}+\lambda\left\langle\Delta Q_{n}(x), \Delta M_{n}^{(\gamma, \mu)}(x)\right\rangle \\
= & k_{n}+\lambda\left\langle\Delta Q_{n}(x), n M_{n-1}^{(\gamma, \mu)}(x)-\frac{n \mu}{1-\mu} \Delta M_{n-1}^{(\gamma, \mu)}(x)\right\rangle \\
= & k_{n}+\lambda n^{2} k_{n-1}-\lambda \frac{n \mu}{1-\mu}\left\langle\Delta Q_{n}(x), \Delta M_{n-1}^{(\gamma, \mu)}(x)\right\rangle \\
= & k_{n}+\lambda n^{2} k_{n-1}-\frac{n \mu}{1-\mu}\left[\left\langle Q_{n}(x), M_{n-1}^{(\gamma, \mu)}(x)\right\rangle_{\mathrm{S}}-\left\langle Q_{n}(x), M_{n-1}^{(\gamma, \mu)}(x)\right\rangle\right] \\
= & k_{n}+\lambda n^{2} k_{n-1}+\frac{n \mu}{1-\mu}\left\langle Q_{n}(x), M_{n-1}^{(\gamma, \mu)}(x)\right\rangle \\
= & k_{n}+\lambda n^{2} k_{n-1}+\frac{n \mu}{1-\mu} \\
& \times\left\langle M_{n}^{(\gamma, \mu)}(x)+\frac{n \mu}{1-\mu} M_{n-1}^{(\gamma, \mu)}(x)-d_{n-1}(\lambda) Q_{n-1}(x), M_{n-1}^{(\gamma, \mu)}(x)\right\rangle \\
= & k_{n}+\lambda n^{2} k_{n-1}+\left(\frac{n \mu}{1-\mu}\right)^{2} k_{n-1}-d_{n-1}(\lambda) \frac{n \mu}{1-\mu} k_{n-1} .
\end{aligned}
$$

Thus, from (24)

$$
d_{n}(\lambda)=\frac{((n+1) \mu /(1-\mu)) k_{n}}{k_{n}+k_{n-1}\left(\lambda n^{2}+(n \mu /(1-\mu))^{2}-(n \mu /(1-\mu)) d_{n-1}(\lambda)\right)}
$$

Finally from (14) we obtain (29), and from (24) we get the initial condition.

Remark 2 Although the coefficients $d_{n}(\lambda)$ appear in the previous results for $n \geq 1$, we can start the recurrence relation (29) with the initial condition $d_{-1}(\lambda)=0$, obtaining the same coefficients for $n \geq 1$. Moreover, for each fixed $n \geq 1$ the coefficient $d_{n}(\lambda)$ is a rational function in $\lambda$ of degree $n-1$ in the numerator and of degree $n$ in the denominator. Thus $\lim _{\lambda \rightarrow \infty} d_{n}(\lambda)=0$ for all $n \geq 1$.

Remark 3 We can write the coefficients $d_{n}(\lambda)$ given by (29) as

$$
\begin{aligned}
d_{n}(\lambda) & =\frac{N_{n-1}(\lambda)}{D_{n}(\lambda)}=\frac{\vartheta_{n}}{\left(\lambda \varpi_{n}+\nu_{n}\right)-\mu(1-\mu)^{2} d_{n-1}(\lambda)} \\
& =\frac{\vartheta_{n}}{\left(\lambda \varpi_{n}+\nu_{n}\right)-\mu(1-\mu)^{2} N_{n-2}(\lambda) / D_{n-1}(\lambda)},
\end{aligned}
$$


where

$$
\begin{gathered}
\varpi_{n}=(1-\mu)^{3} n, \quad \nu_{n}=(1-\mu) \mu(\gamma+n(\mu+1)-1), \\
\vartheta_{n}=\mu^{2}(n+1)(\gamma+n-1),
\end{gathered}
$$

i.e.,

$$
\frac{N_{n-1}(\lambda)}{D_{n}(\lambda)}=\frac{\vartheta_{n} D_{n-1}(\lambda)}{\left(\lambda \varpi_{n}+\nu_{n}\right) D_{n-1}(\lambda)-\mu(1-\mu)^{2} N_{n-2}(\lambda)}
$$

Thus, the denominators $D_{n}(\lambda)$ satisfy the following three-term recurrence relation:

$$
D_{n}(\lambda)=\left(\lambda \varpi_{n}+\nu_{n}\right) D_{n-1}(\lambda)-\mu(1-\mu)^{2} \vartheta_{n-1} D_{n-2}(\lambda), \quad n \geq 2,
$$

which in the monic case, $E_{n}(\lambda)=D_{n}(\lambda) /\left(n !(\mu-1)^{2 n+1}\right)$, can be written

$$
\begin{array}{r}
E_{n}(\lambda)=\left(\lambda-\beta_{n}\right) E_{n-1}(\lambda)-\kappa_{n} E_{n-2}(\lambda), \quad n \geq 2, \\
E_{0}(\lambda)=1, \quad E_{1}(\lambda)=\lambda+\frac{\gamma \mu}{(1-\mu)^{2}},
\end{array}
$$

where

$$
\beta_{n}=\frac{\mu}{(1-\mu)^{2}}\left(\frac{1-\gamma}{n}-(\mu+1)\right), \quad \kappa_{n}=\frac{\mu^{3}(\gamma+n-2)}{(\mu-1)^{4}(n-1)}
$$

If $\left\{P_{n}(x)\right\}_{n}$ is a MOPS satisfying the three-term recurrence relation

$$
P_{n}(x)=\left(x-\phi_{n}\right) P_{n-1}(x)-\psi_{n} P_{n-2}(x), \quad n \geq 2,
$$

then the polynomials $S_{n}(x)=\alpha^{-n} P_{n}(\alpha x+\omega)$, with $\alpha \neq 0$, satisfy [5, p. 25]

$$
S_{n}(x)=\left(x-\frac{\phi_{n}-\omega}{\alpha}\right) S_{n-1}(x)-\frac{\psi_{n}}{\alpha^{2}} S_{n-2}(x), \quad n \geq 2 .
$$


In $\left[5\right.$, p. 187, Eq. (5.18)] we find the Pollaczek polynomials $r_{n}(x$; $a, b, c) \equiv r_{n}(x)$ satisfy the following three-term recurrence relation:

$$
\begin{gathered}
r_{n}(x)=\left(x-\frac{\xi a-(n+a-1) b}{2(n+a)(n+a-1)}\right) r_{n-1}(x) \\
-\frac{(n-1)(n+c-1)}{4(n+a-1)^{2}} r_{n-2}(x), \quad n \geq 2, \\
r_{0}(x)=1, \quad r_{1}(x)=x+\frac{b-\xi}{2(1+a)},
\end{gathered}
$$

where $\xi$ is either a root of $a x^{2}+b x+a-c=0$. If we choose

$$
\begin{aligned}
& \alpha=\frac{(1-\mu)^{2}}{2 \mu^{3 / 2}}, \quad \omega=\frac{\mu+1}{2 \sqrt{\mu}}, \quad a=0, \\
& b=\frac{\gamma-1}{\sqrt{\mu}}, \quad c=\gamma-1, \quad \xi=\sqrt{\mu}
\end{aligned}
$$

then

$$
E_{n}(\lambda)=\alpha^{-n} r_{n}\left(\alpha \lambda+\omega ; 0, \frac{\gamma-1}{\sqrt{\mu}}, \gamma-1\right), \quad n \geq 0,
$$

which are orthogonal with respect to the weight function given in [5, p. 187, Eq. (5.20)]. Moreover, if $\gamma=1$ the coefficients in the threeterm recurrence relation for $E_{n}(\lambda)$ are constant, and $E_{n}(\lambda)$ are co-recursive of monic second kind Chebyshev polynomials $\left\{U_{n}(x)\right\}_{n}$ [5, p. 5]. Therefore $E_{n}(\lambda)$ can be computed explicitly by means of

$$
E_{n}(\lambda)=\alpha^{-n}\left(U_{n}(\alpha \lambda+\omega)-\frac{\mu^{1 / 2}}{2} U_{n-1}(\alpha \lambda+\omega)\right), \quad n \geq 1,
$$

where $\alpha$ and $\omega$ are defined in (30).

Monic Meixner orthogonal polynomials are related with monic Laguerre orthogonal polynomials $\left\{L_{n}^{(\alpha)}(x)\right\}_{n}$ by means of the following limit relation (see [5, p. 177, Eq. (3.8)]):

$$
\lim _{\mu \rightarrow 1}(1-\mu)^{n} M_{n}^{(\alpha+1, \mu)}\left(\frac{x}{1-\mu}\right)=L_{n}^{(\alpha)}(x) .
$$


A limit relation between monic Meixner-Sobolev orthogonal polynomials $\left\{Q_{n}^{(\gamma, \mu)}(x ; \lambda)\right\}_{n}$ and monic Laguerre-Sobolev orthogonal polynomials [21] appears in a natural way.

PROPOSITION 4 The following limit relation holds:

$$
\lim _{\mu \rightarrow 1}(1-\mu)^{n} Q_{n}^{(\alpha+1, \mu)}\left(\frac{x}{1-\mu} ; \frac{\lambda}{(1-\mu)^{2}}\right)=Q_{n}^{(\alpha)}(x), \quad n \geq 1,
$$

where $\left\{Q_{n}^{(\alpha)}(x)\right\}_{n}$ are the Laguerre-Sobolev polynomials [21].

Proof From Eq. (28) we have for $n \geq 2$

$$
\begin{aligned}
(1- & \mu)^{n} Q_{n}^{(\alpha+1, \mu)}\left(\frac{x}{1-\mu} ; \frac{\lambda}{(1-\mu)^{2}}\right) \\
= & (1-\mu)^{n} M_{n}^{(\alpha+1, \mu)}\left(\frac{x}{1-\mu}\right)+n \mu(1-\mu)^{n-1} M_{n-1}^{(\alpha+1, \mu)}\left(\frac{x}{1-\mu}\right) \\
& -(1-\mu) d_{n-1}\left(\frac{\lambda}{(1-\mu)^{2}}\right)(1-\mu)^{n-1} Q_{n-1}^{(\alpha+1, \mu)}\left(\frac{x}{1-\mu} ; \frac{\lambda}{(1-\mu)^{2}}\right) .
\end{aligned}
$$

Since $(1-\mu) d_{n}\left(\lambda /(1-\mu)^{2}\right)$ converges to the coefficients given in [21, Proposition 3.3] when $\mu \rightarrow 1$, the result follows by using the limit relation (32) as well as the equality

$$
Q_{1}^{(\alpha+1, \mu)}\left(\frac{x}{1-\mu} ; \frac{\lambda}{(1-\mu)^{2}}\right)=M_{1}^{(\alpha+1, \mu)}\left(\frac{x}{1-\mu}\right)
$$

\section{THE LINEAR OPERATOR $\mathcal{S}$}

Even the inner product in (15) no longer satisfies the basic property $\langle x p(x), q(x)\rangle_{\mathrm{S}}=\langle p(x), x q(x)\rangle_{\mathrm{S}}$, i.e., $\left\{Q_{n}(x)\right\}_{n}$ does not satisfy a threeterm recurrence relation, this inner product is symmetric with respect to the new operator $\mathcal{S}$.

PROPOSITION 5 If we define the polynomial

$$
h(x)=\mu(x+\gamma-1)
$$


with $0<\mu<1$ and $\gamma>0$, and the linear difference operator $\mathcal{S}$ by

$$
\begin{aligned}
\mathcal{S} & \equiv h(x) \mathcal{I}+\lambda(x-h(x)) \Delta-\lambda x \Delta \nabla \\
& \equiv h(x) \mathcal{I}-\lambda(x \Delta \nabla+(x(\mu-1)+\mu(\gamma-1)) \Delta),
\end{aligned}
$$

where $\mathcal{I}$ is the identity operator, then

$$
\langle h(x) p(x), q(x)\rangle_{\mathrm{S}}=\langle p(x), \mathcal{S} q(x)\rangle
$$

for every polynomial $p$ and $q$.

Proof It is well known (see e.g. [25, p. 21, Eq. (2.1.17)]), that $\rho(s)$ defined in (8) satisfies

$$
\frac{\rho(s+1)}{\rho(s)}=\frac{\sigma(s)+\tau(s)}{\sigma(s+1)}
$$

with $\sigma$ and $\tau$ given in (7). Since $\sigma(s)+\tau(s)=\mu(\gamma+s)$, we obtain

$$
s \rho(s)=\mu(\gamma+s-1) \rho(s-1) .
$$

Now we can compute, by using (6)

$$
\begin{aligned}
& \langle h(x) p(x), q(x)\rangle_{\mathrm{S}} \\
& =\sum_{s=0}^{\infty} h(s) p(s) q(s) \rho(s)+\lambda \sum_{s=0}^{\infty} \Delta(h(s) p(s)) \Delta q(s) \rho(s) \\
& =\sum_{s=0}^{\infty} p(s)(h(s) q(s)-\lambda h(s) \Delta q(s)) \rho(s) \\
& \quad+\lambda \sum_{s=0}^{\infty} h(s+1) p(s+1) \Delta q(s) \rho(s) \\
& =\sum_{s=0}^{\infty} p(s)(h(s) q(s)-\lambda h(s) \Delta q(s)) \rho(s) \\
& \quad+\lambda \sum_{s=1}^{\infty} h(s) p(s) \nabla q(s) \rho(s-1) .
\end{aligned}
$$


By using Eq. (37) we can write the above expression as

$$
\langle h(x) p(x), q(x)\rangle_{\mathrm{S}}=\sum_{s=0}^{\infty} p(s)(h(s) q(s)-\lambda h(s) \Delta q(s)+\lambda s \nabla q(s)) \rho(s)
$$

and from (6) we obtain (36).

Remark 4 Notice that the linear operator $\mathcal{S}$ maps polynomials of exact degree $n$ in polynomials of exact degree $n+1$.

THEOREM 1 The linear operator $\mathcal{S}$ defined in Eq. (35) is symmetric with respect to the Sobolev inner product (15), i.e.,

$$
\langle\mathcal{S} p(x), q(x)\rangle_{\mathrm{S}}=\langle p(x), \mathcal{S} q(x)\rangle_{\mathrm{S}} .
$$

Proof

$$
\begin{aligned}
\langle\mathcal{S} p(x), q(x)\rangle_{\mathrm{S}}= & \sum_{s=0}^{\infty} \mathcal{S} p(s) q(s) \rho(s)+\lambda \sum_{s=0}^{\infty} \Delta(\mathcal{S} p(s)) \Delta q(s) \rho(s) \\
= & \sum_{s=0}^{\infty} \mathcal{S} p(s)(q(s)-\lambda \Delta q(s)) \rho(s) \\
& +\lambda \sum_{s=0}^{\infty} \mathcal{S} p(s+1) \Delta q(s) \rho(s) \\
= & \sum_{s=0}^{\infty} \mathcal{S} p(s)(q(s)-\lambda \Delta q(s)) \hat{\rho}(s) \\
& +\lambda \sum_{s=1}^{\infty} \mathcal{S} p(s) \nabla q(s) \rho(s-1) \\
= & \sum_{s=0}^{\infty} \mathcal{S} p(s)(q(s)-\lambda \Delta q(s)) \rho(s) \\
& +\lambda \sum_{s=1}^{\infty} \mathcal{S} p(s) \nabla q(s) \rho(s-1) \frac{h(s)}{h(s)}
\end{aligned}
$$

since $h(s) \neq 0$ if $s \geq 1$. At this point we must distinguish two situations: If $\gamma \neq 1$ we can write the above expression by using Eq. (37) as

$$
\begin{aligned}
\langle\mathcal{S} p(x), q(x)\rangle_{\mathrm{S}} & =\sum_{s=0}^{\infty} \mathcal{S} p(s)\left(q(s)-\lambda \Delta q(s)+\frac{\lambda s \nabla q(s)}{h(s)}\right) \rho(s) \\
& =\sum_{s=0}^{\infty} \frac{\mathcal{S} p(s) \mathcal{S} q(s)}{h(s)} \rho(s),
\end{aligned}
$$

and this leads to the result. 
Moreover, if $\gamma=1$ then $\rho(s)=\mu^{s}, \mathcal{S} \equiv s(\mu \mathcal{I}+\lambda(1-\mu) \Delta-\lambda \Delta \nabla)$, $\grave{\rho}(s-1)=\rho(s) / \mu$. We can write

$$
\begin{aligned}
\left(\mathcal{S}_{p}(x), q(x)\right)_{\mathrm{S}} & =\sum_{s=1}^{\infty} \mathcal{S} p(s)\left(q(s)-\lambda \Delta q(s)+\frac{\lambda \nabla q(s)}{\mu}\right) \rho(s) \\
& =\sum_{s=1}^{\infty} \frac{\mathcal{S} p(s) \mathcal{S} q(s)}{h(s)} \rho(s),
\end{aligned}
$$

and the resuit holds.

Remark 5 If $\gamma=1$ the difference operator $\mathcal{S}$ reduces to $\mathcal{S} \equiv$ $s(\mu \mathcal{I}+\lambda(1-\mu) \Delta-\lambda \Delta \nabla)$ and we arrive to the analogous situation of Laguerre-Sobolev polynomials with parameter $\alpha=0$ studied by Brenner [4].

Proposition 6 We have

$$
h(x) M_{n}^{(\gamma, \mu)}(x)=\mu Q_{n+1}(x)+a_{n, n} Q_{n}(x)+a_{n-1, n} Q_{n-1}(x), \quad n \geq 2,
$$

where

$$
\begin{aligned}
a_{n, n} & =\mu\left(\frac{\gamma+n-1}{1-\mu}+d_{n}(\lambda)\right), \\
a_{n-1, n} & =\frac{\mu}{1-\mu}(\gamma+n-1) d_{n-1}(\lambda),
\end{aligned}
$$

with $h(x)$ and $d_{n}(\lambda)$ introduced in (34) and (24), respectively.

Proof By using the three-term recurrence relation satisfied by Meixner polynomials ( 9 ) we have

$$
\begin{aligned}
h(x) M_{n}^{(\gamma, \mu)}(x)= & \mu(\gamma+x-1) M_{n}^{(\gamma, \mu)}(x) \\
= & \mu M_{n+1}^{(\gamma, \mu)}(x)+\frac{\mu}{1-\mu}(\gamma+n-1+\mu(n+1)) M_{n}^{(\gamma, \mu)}(x) \\
& +\frac{\mu^{2} n}{(1-\mu)^{2}}(\gamma+n-1) M_{n-1}^{(\gamma, \mu)}(x) \\
= & \mu\left(M_{n+1}^{(\gamma, \mu)}(x)+\frac{\mu}{1-\mu}(n+1) M_{n}^{(\gamma, \mu)}(x)\right) \\
& +\frac{\mu}{1-\mu}(\gamma+n-1)\left(M_{n}^{(\gamma, \mu)}(x)+\frac{n \mu}{1-\mu} M_{n-1}^{(\gamma, \mu)}(x)\right) .
\end{aligned}
$$

Using (21)-(23), the result holds. 
From Propositions 2 and 6, we obtain a non-standard four-term recurrence relation for Meixner-Sobolev orthogonal polynomials.

COROLLARY 3 The Meixner-Sobolev orthogonal polynomials $\left\{Q_{n}(x)\right\}_{n}$ defined in (18) satisfy the following recurrence relation:

$$
\begin{aligned}
& x Q_{n}(x) \\
& =Q_{n+1}(x)+\left(\frac{n+\mu(\gamma+n-1)}{1-\mu}+d_{n}(\lambda)\right) Q_{n}(x) \\
& \quad+\left(\frac{\mu n(\gamma+n-2)-(\mu-1)(n+\mu(\gamma+n-1)) d_{n-1}(\lambda)}{(\mu-1)^{2}}-x d_{n-1}(\lambda)\right) \\
& \quad \times Q_{n-1}(x)+\left(\frac{\mu n(\gamma+n-2) d_{n-2}(\lambda)}{(\mu-1)^{2}}\right) Q_{n-2}(x), \quad n \geq 1,
\end{aligned}
$$

where $d_{n}(\lambda)$ are defined in (24), with the conventions $d_{-1}(\lambda)=0$ and $d_{0}(\lambda)=\mu /(1-\mu)$ and the initial conditions $Q_{-1}(x)=0, Q_{0}(x)=1$ and $Q_{1}(x)=M_{1}^{(\gamma, \mu)}(x)$.

Proof Multiplying Eq. (23) by $h(x)$ and using Eq. (39) we obtain the four-term recurrence relation.

Proposition 7 We have

$$
\mathcal{S} Q_{n}(x)=\mu M_{n+1}^{(\gamma, \mu)}(x)+b_{n, n} M_{n}^{(\gamma, \mu)}(x)+b_{n-1, n} M_{n-1}^{(\gamma, \mu)}(x), \quad n \geq 2
$$

where

$$
\begin{aligned}
b_{n, n} & =\frac{\mu^{2}(n+1)}{(1-\mu)}\left(1+\frac{(\gamma+n-1)}{d_{n}(\lambda)(1-\mu)}\right), \\
b_{n-1, n} & =n(n+1)\left(\frac{\mu}{1-\mu}\right)^{3} \frac{\gamma+n-1}{d_{n}(\lambda)} .
\end{aligned}
$$

Proof If we expand $\mathcal{S} Q_{n}(x)$ in terms of $\left\{\hat{M}_{n}^{(\gamma, \mu)}(x)\right\}_{n}$ we can write

$$
\mathcal{S} Q_{n}(x)=\mu M_{n+1}^{(\gamma, \mu)}(x)+\sum_{i=0}^{n} b_{i, n} M_{i}^{(\gamma, \mu)}(x)
$$


where

$$
b_{i, n}=\frac{\left\langle\mathcal{S} Q_{n}(x), M_{i}^{(\gamma, \mu)}(x)\right\rangle}{k_{i}}=\frac{\left\langle Q_{n}(x), h(x) M_{i}^{(\gamma, \mu)}(x)\right\rangle_{\mathrm{S}}}{k_{i}} .
$$

Hence $b_{i, n}=0$ for $i=0,1, \ldots, n-2$, and

$$
b_{n-1, n}=\frac{\left\langle Q_{n}(x), h(x) M_{n-1}^{(\gamma, \mu)}(x)\right\rangle_{\mathrm{S}}}{k_{n-1}}=\mu \frac{\tilde{k}_{n}}{k_{n-1}}=\mu^{2} \frac{(n+1) k_{n}}{(1-\mu) d_{n}(\lambda) k_{n-1}} .
$$

Finally we can compute

$$
\begin{aligned}
b_{n, n} & =\frac{\left\langle Q_{n}(x), h(x) M_{n}^{(\gamma, \mu)}(x)\right\rangle_{\mathrm{S}}}{k_{n}} \\
& =\frac{\left\langle Q_{n}(x), \mu Q_{n+1}(x)+a_{n, n} Q_{n}(x)+a_{n-1, n} Q_{n-1}(x)\right\rangle_{\mathrm{S}}}{k_{n}} \\
& =a_{n, n} \frac{\tilde{k}_{n}}{k_{n}}
\end{aligned}
$$

\section{Proposition 8 We have}

$$
\mathcal{S} Q_{n}(x)=\mu Q_{n+1}(x)+c_{n, n} Q_{n}(x)+c_{n-1, n} Q_{n-1}(x), \quad n \geq 2,
$$

where

$$
\begin{gathered}
c_{n, n}=\frac{\mu^{2}(n+1)(\gamma+n-1)}{(1-\mu)^{2} d_{n}(\lambda)}+\mu d_{n}(\lambda), \\
c_{n-1, n}=\mu \frac{\tilde{k}_{n}}{\tilde{k}_{n-1}}=\left(\frac{\mu}{1-\mu}\right)^{2}(n+1)(\gamma+n-1) \frac{d_{n-1}(\lambda)}{d_{n}(\lambda)} .
\end{gathered}
$$

Proof If we expand the polynomial $S Q_{n}(x)$ in terms of polynomials $\left\{Q_{n}(x)\right\}_{n}$

$$
\mathcal{S} Q_{n}(x)=\mu Q_{n+1}(x)+\sum_{i=0}^{n} c_{i, n} Q_{i}(x)
$$


by using the symmetric character of the linear operator $\mathcal{S}$ we get

$$
c_{i, n}=\frac{\left\langle\mathcal{S} Q_{n}(x), Q_{i}(x)\right\rangle_{\mathrm{S}}}{\left\langle Q_{i}(x), Q_{i}(x)\right\rangle_{\mathrm{S}}}=\frac{\left\langle Q_{n}(x), S Q_{i}(x)\right\rangle_{S}}{\tilde{k}_{i}} .
$$

Thus $c_{i, n}=0$ for $i=0,1, \ldots, n-2$, and

$$
c_{n-1, n}=\frac{\left\langle Q_{n}(x), \mathcal{S} Q_{n-1}(x)\right\rangle_{\mathrm{S}}}{\tilde{k}_{n-1}}=\mu \frac{\tilde{k}_{n}}{\tilde{k}_{n-1}} .
$$

Finally,

$$
\begin{aligned}
\mathcal{c}_{n, n} & =\frac{\left\langle Q_{n}(x), \mathcal{S} Q_{n}(x)\right\rangle_{\mathrm{S}}}{\tilde{k}_{n}} \\
& =\frac{\left\langle\mu M_{n+1}(x)+b_{n, n} M_{n}(x)+b_{n-1, n} M_{n-1}(x), Q_{n}(x)\right\rangle_{\mathrm{S}}}{\tilde{k}_{n}} \\
& =\mu \frac{\left\langle M_{n+1}(x), Q_{n}(x)\right\rangle_{\mathrm{S}}}{\tilde{k}_{n}}+b_{n, n} .
\end{aligned}
$$

So we must compute $\left\langle M_{n+1}(x), Q_{n}(x)\right\rangle_{\mathrm{S}}$. Since $h(x)=\mu(x+\gamma-1)$ from (9) we get

$$
\begin{aligned}
b_{n, n} & =\frac{\left\langle Q_{n}(x), h(x) M_{n}(x)\right\rangle_{\mathrm{S}}}{k_{n}} \\
& =\mu \frac{\left\langle Q_{n}(x), x M_{n}(x)\right\rangle_{\mathrm{S}}}{k_{n}}+\mu(\gamma-1) \frac{\left\langle Q_{n}(x), M_{n}(x)\right\rangle_{\mathrm{S}}}{k_{n}} \\
& =\mu \frac{\left\langle Q_{n}(x), M_{n+1}(x)\right\rangle_{\mathrm{S}}}{\dot{k}_{n}}+\mu \frac{\tilde{k}_{n}}{k_{n}}\left(B_{n}+\gamma-1\right),
\end{aligned}
$$

where $B_{n}$ is given in (10). So we obtain

$$
\begin{aligned}
\mu \frac{\left\langle Q_{n}(x), M_{n+1}(x)\right\rangle_{S}}{\tilde{k}_{n}} & =\left(b_{n, n}-\mu \frac{\tilde{k}_{n}}{k_{n}}\left(B_{n}+\gamma-1\right)\right) \frac{k_{n}}{\tilde{k}_{n}} \\
& =a_{n, n}-\mu\left(B_{n}+\gamma-1\right),
\end{aligned}
$$

and then the result holds. 


\section{ZEROS}

It is well known that the zeros of Meixner polynomials $M_{n}^{(\gamma, \mu)}(x)$ are real and distinct. They also lie on the interval of orthogonality $[0,+\infty)$ and they separate the zeros of $M_{n-1}^{(\gamma, \mu)}(x)$ (see [26] and the notes of Fejèr at the end of [12]). In this section we study the location of the zeros of Meixner-Sobolev orthogonal polynomials $\left\{Q_{n}(x)\right\}_{n}$ and an interlacing property which relates the zeros of $Q_{n}(x)$ to the zeros of $M_{n}^{(\gamma, \mu)}(x)$.

LEMMA 1 If $n \geq 0$ and if $\gamma \geq 1$ we have $(-1)^{n} Q_{n}(0)>0$.

Proof We shall prove that $Q_{n}(0)$ and $M_{n}^{(\gamma, \mu)}(0)$ have the same sign or, equivalently, $Q_{n}(0) / M_{n}^{(\gamma, \mu)}(0)>0$ for all $n \geq 0$. Then, by using the value of $M_{n}^{(\gamma, \mu)}(0)$ given in Eq. (12) the result follows since $0<\mu<1$.

If we write Eq. (28) for $x=0$ we obtain a recurrence relation for $Q_{n}(0):$

$$
\begin{gathered}
Q_{n}(0)=M_{n}^{(\gamma, \mu)}(0)+\frac{n \mu}{1-\mu} M_{n-1}^{(\gamma, \mu)}(0)-d_{n-1}(\lambda) Q_{n-1}(0), \quad n \geq 2, \\
Q_{1}(0)=M_{1}^{(\gamma, \mu)}(0) .
\end{gathered}
$$

By using Eq. (12) we get

$$
\begin{aligned}
& M_{n}^{(\gamma, \mu)}(0)+\frac{n \mu}{1-\mu} M_{n-1}^{(\gamma, \mu)}(0) \\
& \quad=\left(\frac{\mu}{\mu-1}\right)^{n}(\gamma)_{n}+\frac{n \mu}{1-\mu}\left(\frac{\mu}{\mu-1}\right)^{n-1}(\gamma)_{n-1} \\
& \quad=(\gamma-1) \frac{\mu}{\mu-1} M_{n-1}^{(\gamma, \mu)}(0)
\end{aligned}
$$

Thus, from the above equation we can write Eq. (49) as

$$
Q_{n}(0)=(\gamma-1) \frac{\mu}{\mu-1} M_{n-1}^{(\gamma, \mu)}(0)-d_{n-1}(\lambda) Q_{n-1}(0) .
$$

From Eq. (12) we can also deduce

$$
M_{n}^{(\gamma, \mu)}(0)=\frac{\mu}{\mu-1}(\gamma+n-1) M_{n-1}^{(\gamma, \mu)}(0) .
$$


So, taking into account the last expression we get

$$
\begin{aligned}
\frac{Q_{n}(0)}{M_{n}^{(\gamma, \mu)}(0)} & =(\gamma-1) \frac{\mu}{\mu-1} \frac{M_{n-1}^{(\gamma, \mu)}(0)}{M_{n}^{(\gamma, \mu)}(0)}-d_{n-1}(\lambda) \frac{Q_{n-1}(0)}{M_{n}^{(\gamma, \mu)}(0)} \\
& =\frac{\gamma-1}{\gamma+n-1}-d_{n-1}(\lambda) \frac{(\mu-1)}{\mu(\gamma+n-1)} \frac{Q_{n-1}(0)}{M_{n-1}^{(\gamma ; \mu)}(0)}
\end{aligned}
$$

If we denote $\mathcal{A}_{n}=Q_{n}(0) / M_{n}^{(\gamma, \mu)}(0)$ the above expression can be written

$$
\mathcal{A}_{n}=\frac{\gamma-1}{\gamma+n-1}-d_{n-1}(\lambda) \frac{(\mu-1)}{\mu(\gamma+n-1)} \mathcal{A}_{n-1}
$$

Finally, from Eq. (24) we obtain

$$
\begin{aligned}
& \mathcal{A}_{1}=\frac{Q_{1}(0)}{M_{1}^{(\gamma, \mu)}(0)}=1, \\
& \mathcal{A}_{n}=\frac{1}{\gamma+n-1}\left((\gamma-1)+n \frac{k_{n-1}}{\tilde{k}_{n-1}} \mathcal{A}_{n-1}\right), \quad n \geq 2 .
\end{aligned}
$$

Since $k_{1} / \tilde{k}_{1} \geq 0, \mathcal{A}_{2}$ is positive for $\gamma \geq 1$, and then $\mathcal{A}_{n}>0$ for all $n \geq 1$. Thus $\operatorname{sgn}\left(Q_{n}(0)\right)=\operatorname{sgn}\left(M_{n}^{(\gamma, \mu)}(0)\right)=\operatorname{sgn}\left((\mu-1)^{n}\right)$, for all $n \geq 1$, so we get $Q_{2 k}(0)>0$ and $Q_{2 k+1}(0)<0$. The case $n=0$ follows from $Q_{0}(0)=M_{0}^{(\gamma, \mu)}(0)=1$.

LEMMA 2 Let $p(x)$ be a polynomial of degree $k$. If $\lambda=0$ or $\gamma=1$ there exists a unique polynomial $p_{1}(x)$ of degree $k$ such that $\mathcal{S}_{1}(x)=h(x) p(x)$, where $S$ and $h(x)$ are defined in Proposition 5.

Proof If $\lambda=0$ the linear operator $\mathcal{S}$ becomes $\mathcal{S} \equiv h(x) \mathcal{I}$, where $\mathcal{I}$ stands for the identity operator. Then it is sufficient to take $p_{1}(x)=p(x)$.

If $\gamma=1$, the linear operator $\mathcal{S}$ can be written

$$
\mathcal{S} \equiv \mu x \mathcal{I}+\lambda(1-\mu) x \Delta-\lambda x \Delta \nabla .
$$

Let us expand

$$
p_{1}(x)=\sum_{i=0}^{k} b_{i}(x+1)^{[i]}, \quad h(x) p(x)=\mu x p(x)=\sum_{i=0}^{k+1} a_{i} x^{[i]} .
$$


The following basic properties will be useful in the proof:

$$
\begin{gathered}
x x^{[m]}=x^{[m+1]}+m x^{[m]}, \quad \Delta x^{[m]}=m x^{[m-1]}, \quad \nabla x^{[m]}=m(x-1)^{[m-1]}, \\
h(x)(x+1)^{[m]}=\mu\left(x^{[m+1]}+2 m x^{[m]}+m(m-1) x^{[m-1]}\right), \\
\lambda h(x) \Delta(x+1)^{[m]}=\lambda m \mu\left(x^{[m]}+2(m-1) x^{[m-1]}\right. \\
\left.+(m-1)(m-2) x^{[m-2]}\right), \\
\lambda x \Delta x^{[m]}=\lambda m\left(x^{[m]}+(m-1) x^{[m-1]}\right) .
\end{gathered}
$$

Hence, the action of the operator $\mathcal{S}$ on $p_{1}(x)$ yields

$$
\begin{aligned}
S p_{1}(x)= & \sum_{i=0}^{n} b_{i}\left(\mu x^{[i+1]}+i(\lambda+\mu(2-\lambda)) x^{[i]}\right. \\
& \left.+i(i-1)(\lambda+\mu(1-2 \lambda)) x^{[i-1]}-i(i-1)(i-2) \lambda \mu x^{[i-2]}\right) .
\end{aligned}
$$

From the equality $\mathcal{S}_{\bar{p}}(x)=h(x) p(x)$ we obtaini a systeni of $(k+1)$ linear equations with $(k+1)$ unknowns. It has a unique solution which can be obtained using the forward substitution method.

COROLlary 4 Let $p(x)$ be a polynomial of degree $k$ and assume $\gamma=1$. Let $p_{1}(x)$ be the polynomial of degree $k$ such that $S p_{1}(x)=h(x) p(x)$. Then

$$
\left\langle q(x), p_{1}(x)\right\rangle_{\mathrm{S}}=\langle q(x), p(x)\rangle-\lambda \frac{q(0) \nabla p_{1}(0)}{\mu}, \quad \forall q(x) \in \mathbb{P} .
$$

\section{Proof We have}

$$
\begin{aligned}
\left\langle q(x), p_{1}(x)\right\rangle_{\mathrm{S}}=\sum_{s=0}^{\infty} q(s)\left(p_{1}(s)-\lambda \Delta p_{1}(s)\right) \rho(s)+\lambda \sum_{s=0}^{\infty} q(s+1) \Delta p_{1}(s) \rho(s) \\
=\sum_{s=0}^{\infty} q(s)\left(p_{1}(s)-\lambda \Delta p_{1}(s)\right) \rho(s)+\lambda \sum_{s=1}^{\infty} q(s) \nabla p_{1}(s) \rho(s-1) \\
=\sum_{s=0}^{\infty} q(s)\left(p_{1}(s)-\lambda \Delta p_{1}(s)\right) \rho(s)+\lambda \sum_{s=1}^{\infty} \frac{q(s) \nabla p_{1}(s) \rho(s)}{\mu} \\
=\sum_{s=0}^{\infty} q(s)\left(p_{1}(s)-\lambda \Delta p_{1}(s)+\lambda \frac{\nabla p_{1}(s)}{\mu}\right) \rho(s)-\lambda \frac{q(0) \nabla p_{1}(0)}{\mu} \\
=\sum_{s=0}^{\infty} q(s) \frac{\mathcal{S} p_{1}(s)}{\mu s} \rho(s)-\lambda \frac{q(0) \nabla p_{1}(0)}{\mu} \\
=\sum_{s=0}^{\infty} q(s) p(s) \rho(s)-\lambda \frac{q(0) \nabla p_{1}(0)}{\mu} \\
=\langle q(x), p(x)\rangle-\lambda \frac{q(0) \nabla p_{1}(0)}{\mu}
\end{aligned}
$$


LEMMA 3 Let $p(x)$ be a polynomial of degree $k$. If $\lambda \neq 0$ and if $\gamma \neq 1$ there exists a unique polynomial $p_{1}(x)$ of degree $k$ as well as a unique constant $c_{p}$ (depending on $p$ ) such that

$$
\mathcal{S}_{p_{1}}(x)=h(x)\left(p(x)+(x+\gamma-2) c_{p}\right) .
$$

Proof Let us write the polynomial $\bar{p}_{1}(x)$ in terms of the basis $\left\{(x+\gamma)^{[i]}\right\}_{i}$ as

$$
p_{1}(x)=\sum_{i=0}^{k} b_{i}(x+\gamma)^{[i]} .
$$

For such a basis we have

$$
\Delta(x+\gamma)^{[i]}=i(x+\gamma)^{[i-1]}, \quad \nabla(x+\gamma)^{[i]}=i(x+\gamma-1)^{[i-1]}
$$

Hence, if we apply $\mathcal{S}$ to any element of the above basis we obtain

$$
\mathcal{S}(x+\gamma)^{[i]} \equiv h(x)(x+\gamma)^{[i]}=\lambda i h(x)(x+\gamma)^{[i-1]}+\lambda i(x+\gamma)^{[i]},
$$

or, equivalently,

$$
\begin{aligned}
\mathcal{S}(x+\gamma)^{[i]}= & \mu(x+\gamma-1)^{[i+1]}+(x+\gamma-1)^{[i]}(i(\lambda+\mu(2-\lambda))) \\
& +(x+\gamma-1)^{[i-1]}(\mu i(i-1)-2 \lambda \mu i(i-1)+\lambda i(i-\gamma)) \\
& -(x+\gamma-1)^{[i-2]} \lambda \mu i(i-1)(i-2) .
\end{aligned}
$$

Let us expand the polynomial $h(x) p(x)$ in the basis $\left\{(x+\gamma-1)^{[i]}\right\}_{i}$ :

$$
h(x) p(x)=\sum_{i=0}^{k+1} a_{i}(x+\gamma-1)^{[i]} .
$$

From $S p_{1}(x)=h(x)\left(p(x)+(x+\gamma-2) c_{p}\right)$ we obtain the following system of $(k+2)$ linear equations with $(k+2)$ unknowns:

$$
\begin{gathered}
\mu b_{k}=a_{k+1}, \\
\mu b_{k-1}+k(\lambda+\mu(2-\lambda)) b_{k}=a_{k},
\end{gathered}
$$




$$
\begin{aligned}
& \mu b_{k-2}+(k-1)(\lambda+\mu(2-\lambda)) b_{k-1}+k(\mu(k-1)(1-2 \lambda) \\
& +\lambda(k-\gamma)) b_{k}=a_{k-1}, \\
& \mu b_{i-3}+(i-2)(\lambda+\mu(2-\lambda)) b_{i-2}+(i-1)(\mu(i-2)(1-2 \lambda) \\
& +\lambda(i-\gamma-1)) b_{i-1}-\lambda \mu i(i-1)(i-2) b_{i}=a_{i-2}, \quad i=k, \ldots, 5 \\
& \mu b_{1}+2(\lambda+\mu(2-\lambda)) b_{2}+3(\mu(2)(1-2 \lambda) \\
& +\lambda(4-\gamma-1)) b_{3}-24 \lambda \mu b_{4}=a_{2}+c_{p}, \\
& \mu \bar{b}_{0}+(\lambda+\mu(2-\lambda)) b_{1}+2(\mu(1-2 \lambda) \\
& +\lambda(3-\gamma-1)) b_{2}-6 \lambda \mu b_{3}=a_{1} \\
& \quad \lambda(1-\gamma) b_{1}=a_{0} .
\end{aligned}
$$

This linear system has a unique solution $\left(b_{0}, b_{1}, \ldots, b_{k}, c_{p}\right)$ which can be found using the forward substitution method. Moreover, if $\gamma \neq 1$ and $\lambda \neq 0$ then $b_{1}=0$ since $a_{0}=0$.

CoROLlaRY 5 Let $p(x)$ be a polynomial of degree $k$ and let $p_{1}(x)$ be the polynomial of degree $k$ and $c_{p}$ be the constant given in the previous lemma such that Eq. (51) is verified. Then

$$
\left\langle q(x), p_{1}(x)\right\rangle_{\mathrm{S}}=\langle q(x), p(x)\rangle+c_{p}\langle x+\gamma-2, q(x)\rangle, \quad \forall q \in \mathbb{P} .
$$

\section{Proof We have}

$$
\begin{aligned}
& \left\langle q(x), p_{1}(x)\right\rangle_{\mathrm{S}} \\
& \quad=\sum_{s=0}^{\infty} q(s) p_{1}(s) \rho(s)+\lambda \sum_{s=0}^{\infty} \Delta q(s) \Delta p_{1}(s) \rho(s) \\
& \quad=\sum_{s=0}^{\infty} q(s)\left(p_{1}(s)-\lambda \Delta p_{1}(s)\right) \rho(s)+\lambda \sum_{s=0}^{\infty} q(s+1) \Delta p_{1}(s) \rho(s) \\
& =\sum_{s=0}^{\infty} q(s)\left(p_{1}(s)-\lambda \Delta p_{1}(s)\right) \rho(s)+\lambda \sum_{s=1}^{\infty} q(s) \nabla p_{1}(s) \rho(s-1) \\
& =\sum_{s=0}^{\infty} q(s)\left(p_{1}(s)-\lambda \Delta p_{1}(s)\right) \rho(s)+\lambda \sum_{s=1}^{\infty} q(s) \nabla p_{1}(s) \frac{h(s)}{h(s)} \rho(s-1) \\
& =\sum_{s=0}^{\infty} q(s)\left(p_{1}(s)-\lambda \Delta p_{1}(s)\right) \rho(s)+\lambda \sum_{s=1}^{\infty} q(s) \nabla p_{1}(s) \frac{s}{h(s)} \rho(s),
\end{aligned}
$$


where the last equality is a consequence of (37). Thus

$$
\begin{aligned}
& \left\langle q(x), p_{1}(x)\right\rangle_{s} \\
& \quad=\sum_{s=0}^{\infty} q(s)\left(p_{1}(s)-\lambda \Delta p_{1}(s)+\frac{\lambda s}{h(s)} \bar{\nabla} p_{1}(s)\right) \rho(s) \\
& \quad=\sum_{s=0}^{\infty} q(s) \frac{\mathcal{S}_{p_{1}}(s)}{h(s)} \rho(s)=\sum_{s=0}^{\infty} q(s) \frac{h(s)\left(p(s)+(s+\gamma-2) c_{p}\right)}{h(s)} \rho(s) \\
& =\langle q(x), p(x)\rangle+c_{p}\langle x+\gamma-2, q(x)\rangle .
\end{aligned}
$$

LEMMA 4 Let $p$ be a polynomial of degree $n$. If $\lambda \neq 0$ and $\gamma \neq 1$ then we have $(-1)^{n} c_{p}>0$, where $c_{p}$ is the constant obtained for $p(x)$ by using Lemma 3.

Proof If we write Eq. (52) for $q(x)=Q_{n}(x)$ it follows

$$
\left\langle Q_{n}(x), p_{1}(x)\right\rangle_{\mathrm{S}}=\left\langle Q_{n}(x), p(x)\right\rangle+c_{p}\left\langle x+\gamma-2, Q_{n}(x)\right\rangle .
$$

From Eq. (27) the above equation reads

$$
\left\langle Q_{n}(x), p_{1}(x)\right\rangle_{S}=\left\langle Q_{n}(x), p(x)\right\rangle+c_{p} e_{1, n} k_{1}=k_{n}+c_{p} e_{1, n} k_{1} .
$$

If $p$ is a monic polynomial of degree $n$, then $p_{1}$ is also a monic polynomial of degree $n$. Thus $\tilde{k}_{n}=k_{n}+c_{p} e_{1, n} k_{\mathrm{l}}$, or, equivalently,

$$
c_{p}=\frac{\tilde{k}_{n}-k_{n}}{e_{1, n} k_{1}} \text {. }
$$

The coefficient $e_{1, n}$ can be computed from (27) and (24):

$$
e_{1, n}=(-1)^{n-2} \frac{2 \lambda \mu(1-\mu)}{\lambda(1-\mu)^{2}+\gamma \mu} \prod_{s=2}^{n-1} \frac{(s+1) \mu}{1-\mu} \frac{\tilde{k}_{s}}{k_{s}},
$$

so $\operatorname{sgn}\left(e_{1, n}\right)=(-1)^{n-2}$.

Finally, from $\tilde{k}_{n}>k_{n}$ it follows that $\operatorname{sgn}\left(c_{p}\right)=(-1)^{n-2}$ and then $(-1)^{n} c_{p}>0$.

THEOREM 2 For each $\lambda>0$ the polynomial $Q_{n}(x), n \geq 2$, has exactly $n$ real and distinct zeros, where at least $n-1$ of them are positive. 
Moreover, if $\gamma \geq 1$ then all the zeros and positive. If we denote by $\dot{x}_{n, 1}<x_{n, 2}<\cdots<x_{n, n}$ the zeros of $M_{n}^{(\gamma, \mu)}(x)$ and if we denote by $y_{n, 1}<y_{n, 2}<\cdots<y_{n, n}$ the $n$ different zeros of $Q_{n}(x)$ then

$$
y_{n, 1}<x_{n, 1}<y_{n, 2}<x_{n, 2}<\cdots<y_{n, n}<x_{n, n}
$$

Proof Let $x_{n, 1}<x_{n, 2}<\cdots<x_{n, n}$ be the zeros of $M_{n}^{(\gamma, \mu)}(x)$ and let us define

$$
w_{i}(x)=\prod_{j=1, j \neq i}^{n}\left(x-x_{n, j}\right) .
$$

- If $\gamma \neq 1$ by using Lemma 3 we obtain a unique polynomial $p_{i}(x)$ of degree $n-1$ as well as a constant $c_{i}$ such that $\mathcal{S} p_{i}(x)=$ $h(x)\left(w_{i}(x)+(x+\gamma-2) c_{i}\right)$. From Corollary 5 we get

$$
\begin{aligned}
0 & =\left\langle Q_{n}(x), p_{i}(x)\right\rangle_{\mathrm{S}}=\left\langle w_{i}(x), Q_{n}(x)\right\rangle+c_{i}\left\langle x+\gamma-2, Q_{n}(x)\right\rangle \\
& =\sum_{s=0}^{\infty} w_{i}(s) Q_{n}(s) \rho(s)+c_{i} \sum_{s=0}^{\infty}(s+\gamma-2) Q_{n}(s) \rho(s) .
\end{aligned}
$$

The Gaussian type quadrature formula based in the zeros of $M_{n}^{(\gamma, \mu)}(x)[25]$ leads to

$$
\begin{aligned}
0 & =\left\langle Q_{n}(x), p_{i}(x)\right\rangle_{S} \\
& =\lambda_{i} w_{i}\left(x_{n, i}\right) Q_{i}\left(x_{n, i}\right)+c_{i} \sum_{s=0}^{\infty}(s+\gamma-2) Q_{n}(s) \rho(s) .
\end{aligned}
$$

Let us compute the second term of the above sum by using (27),

$$
\begin{aligned}
c_{i} \sum_{s=0}^{\infty}(s+\gamma-2) Q_{n}(s) \rho(s) & =c_{i}\left\langle x+\gamma-2, Q_{n}(x)\right\rangle \\
& =c_{i} \sum_{j=1}^{n} e_{j, n}\left\langle x+\gamma-2, M_{j}^{(\gamma, \mu)}(x)\right\rangle=c_{i} e_{1, n} k_{1} .
\end{aligned}
$$

Hence the sign of this second term is always negative since $k_{1}>0$, $\operatorname{sgn}\left(e_{1, n}\right)=(-1)^{n-2}$ and $\operatorname{sgn}\left(c_{i}\right)=(-1)^{n-1}$ (from Lemma 4 because $\left.\operatorname{deg} w_{i}(x)=n-1\right)$. 
Thus from Eq. (55) we deduce

$$
\lambda_{i} w_{i}\left(x_{n, i}\right) Q_{n}\left(x_{n, i}\right)=-c_{i} \sum_{s=0}^{\infty}(s+\gamma-2) Q_{n}(s) \rho(s)>0
$$

and then $Q_{n}\left(x_{n, i}\right) \neq 0$. Moreover $\operatorname{sgn}\left(Q_{n}\left(x_{n, i}\right)\right)=\operatorname{sgn}\left(w_{i}\left(x_{n, i}\right)\right)=(-1)^{n-i}$, so $Q_{n}(x)$ changes sign between two consecutive zeros of $M_{n}^{(\gamma, \mu)}(x)$.

Finally, since $\operatorname{sgn}\left(Q_{n}\left(x_{n,}\right)\right)=(-1)^{n-1}$ then $Q_{n}(x)$ has $n$ different real zeros, which separate those of $M_{n}^{(\gamma, \mu)}(x)$ as stated.

- If $\gamma=1$, we use the orthogonality of $Q_{n}(x)$, Corollary 5 and the Gaussian type quadrature formula for evaluating sums in order to obtain

$$
\begin{aligned}
0=\left\langle Q_{n}(x), p_{i}(x)\right\rangle_{\mathrm{S}} & =\left\langle Q_{n}(x), w_{i}(x)\right\rangle-\lambda \frac{Q_{n}(0) \nabla p_{i}(0)}{\mu} \\
& =\lambda_{i} w_{i}\left(x_{n, i}\right) Q_{n}\left(x_{n, i}\right)-\lambda \frac{Q_{n}(0) \nabla \bar{p}_{i}(0)}{\mu},
\end{aligned}
$$

where $w_{i}(x)$ are the polynomials defined in (54).

In this case we have $\lambda_{i} w_{i}\left(x_{n, i}\right) Q_{n}\left(x_{n, i}\right)=(\lambda / \mu) Q_{n}(0) \nabla p_{i}(0)$. From Lemma 1 we have $(-1)^{n} Q_{n}(0)>0$, and repeating the arguments in Lemma 4 we can obtain $(-1)^{n-2} \nabla p_{i}(0)>0$. Hence we obtain $\lambda_{i} w_{i}\left(x_{n, i}\right) Q_{n}\left(x_{n, i}\right)>0$, and then $Q_{n}\left(x_{n, i}\right) \neq 0$ as well as $\operatorname{sgn}\left(Q_{n}\left(x_{n, i}\right)\right)=$ $(-1)^{n-i}$. The proof follows in the same way as in the case already discussed.

Finally, if $\gamma \geq 1$ we have $(-1)^{n} Q_{n}(0)>0$, using Lemma 1 , so we can deduce that all zeros are positive.

\section{Acknowledgements}

The work of I.A. and E.G. has been partially supported by Xunta de Galicia-Universidade de Vigo under grant 64502I703. E.G. also wishes to acknowledge partial support by Dirección General de Enseñanza Superior (DGES) of Spain under grant PB-95-0828. The research of F.M. was partially supported by DGES of Spain under Grant PB96-0120-C03-01 and INTAS Project 93-0219 Ext. 


\section{References}

[1] M. Alfaro, F. Marcellán, M.L. Rezola and A. Ronveaux, On orthogonal polynomials of Sobolev type: Algebraic properties and zeros, SIAM J. Math. Anal. 23(3) (1992), 737-757.

[2] I. Area, E. Godoy and F. Marcelián, Coherent pairs and orthogonal polynomials of a discrete variable (submitted).

[3] I. Area, E. Godoy, A. Ronveaux and A. Zarzo, Minimal recurrence relations for connection coefficients between classical orthogonal polynomials: discrete case, J. Comput. Appl. Math. 89(2) (1998), 309-325.

[4] J. Brenner, Uber eine Erweiterung des Orthogonalitätsbegriffes bei Polynomen, in: G. Alexits and S.B. Stechkin (eds.), Proc. Conf. on the Constructive Theory of Functions (Budapest, 1969) (Akadémiai Kiadó, Budapest, 1972), pp. 77-83

[5] T.S. Chihara, An Introduction to Orthogonal Polynomials Gordon and Breach, New York, 1978.

[6] E.A. Cohen, Zero distribution and behaviour of orthogonal polynomials in the Sobolev space $W^{1.2}[-1,1]$, SIAM J. Math. Anal. 6(1) (1975), $105-116$

[7] M.G. de Bruin and H.G. Meijer, Zeros of orthogonal polynomials in a non-discrete Sobolev space, Ann. Num. Math. 2 (1995), 233-246.

[8] W.D. Evans, L.L. Littlejohn, F. Marcellán, C. Markett and A. Ronveaux, On recurrence relations for Sobolev orthogonal polynomials, SIAM J. Math. Anal. 26(2) (1995), 446-467.

[9] A.G. García, F. Marcellán and L. Salto, A distributional study of discrete classical orthogonal polynomials, I. Comp. Appl. Math. 57 (1995), 147-162.

[10] W. Gautschi and M. Zhang, Computing orthogonal polynomials in Sobolev spaces, Numer. Math. 71(2) (1995), 159-183.

[11] A. Iserles, P.E. Koch, S.P. Nørsett and J.M. Sanz-Serna, On polynomials orthogonal with respect to certain Sobolev inner products, J. Approximation Theory 65(2) (1991), 151-175.

[12] C. Jordan, Sur une série de polynômes dont chaque somme partielle représente la meilieure approximation d'un degré donné suivant la méthode dés inoindies cancés, Proceedings of the London Mathematical Society 20 (1921), 297-325.

[13] P.E. Koch, An extension of the theory of orthogonal polynomials and Gaussian quadrature to trigonometric and hyperbolic polynomials, J. Approximation Theory 43 (1985), 157-177.

[14] R. Koekoek and R.F. Swarttouw, The Askey-scheme of hypergeometric orthogonal polynomials and its $q$-analogue, TWI Report 94-05. TU Delft, 1994.

[15] D.C. Lewis, Polynomial least square approximations, Amer. J. Math. 69 (1947), $273-278$

[16] F. Marcellán, M. Alfaro and M.L. Rezola, Orthogonal polynomials on Sobolev spaces: old and new directions, J. Comp. Appl. Math. 48 (1993), 113-131.

[17] F. Marcellán and J.C. Petronilho, Orthogonal polynomials and coherent pairs: the classical case, Indag. Math. New Ser. 6(3) (1995), 287-307.

[18] F. Marcellán, J.C. Petronilho, T.E. Pérez and M.A. Piñar, What is beyond coherent pairs of orthogonal polynomials?, J. Comp. Appl. Math. 65 (1995), 267-277.

[19] F. Marcellán, T.E. Pérez and M.A. Piñar, Orthogonal polynomials on weighted Sobolev spaces: the semiclassical case, Ann. Numer. Math. 2 (1995), 93-122.

[20] F. Marcellán, T.E. Pérez and M.A. Piñar. On zeros of Sobolev-type orthogonal polynomials Rend. Mat. Appl., VII, 12(2) (1992), 455-473.

[21] F. Marcellán, T.E. Pérez and M.A. Piñar, Laguerre-Sobolev orthogonal polynomials, J. Comp. Appl. Math. 71 (1996), 245-265.

[22] H.G. Meijer, Determination of all coherent pairs, J. Approximation Theory 89(3) (1997), 321-343. 
[23] H.G. Meijer, A short history of orthogonal polymomials in a Sobolev space. I: The non-discrete case, Nieuw Arch. Wiskd. 14(1) (1996), 93-113.

[24] J. Meixner, Orthogonale Polynomsysteme mit einer besonderen gestalt der erzeugenden Funktionen, J. London Math. Soc. 9 (1934), 6-13.

[25] A.F. Nikiforov, S.K. Suslov and V.B. Uvarov, Classical Orthogonal Polynomials of a Discrete Variable, Springer-Verlag, Berlin, 1991.

[26] M.B. Porter, On the roots of functions connected by a linear recurrent relation of the second order, Annals of Mathematics (2nd series) 3 (1901-02), 55-70. 